\title{
LncRNA XIST acts as a ceRNA sponging miR-185-5p to modulate pancreatic cancer cell proliferation via targeting CCND2
}

\author{
Ya-Peng Wang", Yan Huang", Tao Hou, Min Lu \\ Department of Oncology, Second Xiangya Hospital, Central South University, Changsha 410011, China \\ Contributions: (I) Conception and design: YP Wang; (II) Administrative support: YP Wang, Y Huang; (III) Provision of study materials or patients: T \\ Hou; (IV) Collection and assembly of data: M Lu; (V) Data analysis and interpretation: Y Huang; (VI) Manuscript writing: All authors; (VII) Final \\ approval of manuscript: All authors. \\ \#These authors contributed equally to this work as co-first authors. \\ Correspondence to: Dr. Ya-Peng Wang. Department of Oncology, Second Xiangya Hospital, Central South University, Changsha 410011, China. \\ Email: wyp2008420@csu.edu.cn.
}

Background: Long non-coding RNAs (lncRNAs) have been proved to be involved in the occurrence and progression of various tumors including pancreatic cancer (PC). Growing evidence shows that lncRNA $\mathrm{X}$ inactive-specific transcript (XIST) functions as an oncogene in multiple tumorigenesis. However, the underlying mechanism of lncRNA XIST in the progression of PC remains elusive.

Methods: Expression levels of XIST and miR-185-5p both in PC tissues or PC cells were determined using real-time quantitative PCR (qRT-PCR). Gain and loss-of-function of XIST or miR-185-5p was performed for further exploration. Moreover, colony formation assay was performed to assess cell proliferation. Flow cytometry analysis was performed to measure cell cycle and apoptosis. Dual-luciferase reporter assay was conducted to verify the correlation between XIST, miR-185-5p and CCND2, respectively. Additionally, western blot analysis was conducted to determine the expression pattern of apoptosis-related proteins and cell cycle-associated proteins.

Results: Herein, we found that XIST expression was up-regulated while miR-185-5p was down-regulated both in PC tissues and cell lines, compared with that of controls. Moreover, there was a negative correlation between XIST and miR-185-5p. Following that, functional experiments displayed that knockdown of XIST or overexpression of miR-185-5p inhibited cell proliferation, induced cell cycle arrest and promoted apoptosis in PC cells. Furthermore, mechanistic experiments displayed that XIST could negatively regulate miR-185-5p via direct binding. In addition, CCND2 was shown to be a downstream target of miR-185-5p. Importantly, overexpression or knockdown of XIST significantly increased or decreased the expression of CCND2, while these effects were reversed by miR-185-5p.

Conclusions: Taken together, our study demonstrated that lncRNA XIST functions as an oncogene and exerts its regulation via miR-185-5p/CCND2 axis, promoting proliferation and inhibiting apoptosis in PC.

Keywords: Pancreatic cancer (PC); long non-coding RNAs (lncRNA); X inactive-specific transcript (XIST); miR185-5p; CCND2; proliferation

Submitted May 29, 2019. Accepted for publication Dec 13, 2019.

doi: $10.21037 /$ tcr.2020.01.26

View this article at: http://dx.doi.org/10.21037/tcr.2020.01.26

\section{Introduction}

Due to a high tendency for aggressive invasion and distant metastasis, the lethality rate of pancreatic cancer (PC) is the fourth highest among all cancers (1). It is reported that PC has a high incidence with $>40,000$ cases diagnosed each year and a very low 5 -year survival rate estimated to be $<5 \%(2,3)$. Despite the advancement of surgical technologies including 
chemotherapy and radiotherapy, the efficient remission of PC treatment has not been emerged. Therefore, finding novel biomarkers for identifying biological characteristics of $\mathrm{PC}$ is urgent to further explore.

Long non-coding RNAs (lncRNAs), the RNA without the function of protein coding, consist of more than 200 of nucleotide units (4). Emerging evidence has shown the crucial roles of lncRNAs in various bioprocesses and diseases, not only participate in epigenetic modification and transcription of normal cell, but also regulate the proliferation, differentiation and metastasis of cancer cells (5). It has been demonstrated that the dysregulation of lncRNAs can be potentially functioned as important regulators in the development and progression of PC (6). For example, lncRNA UCA1 was considered to impact the proliferation, invasion and migration of $\mathrm{PC}$ via regulating miR-96/FOXO3 (7). And knockdown of MEG3 enhanced the $\mathrm{PC}$ cell proliferation, migration and invasion, as well as induce epithelial-mesenchymal transition (EMT) (4).

$\mathrm{X}$ inactive-specific transcript (XIST), one of the lncRNA affecting the expression of gene, can mediate the transcription silence of $\mathrm{X}$ chromosome via a complex mechanism (8). Besides, XIST has been proved to be associated with development of multiple cancers including non-small cell lung cancer (NSCLC), gastric cancer, and hematoma (9-11). Furthermore, XIST was also found to be elevated and could enhance the proliferation PC cell via interacting with miR-40/miR-124/iASPP or miR-133a/ EGFR $(12,13)$. However, deeper insights for XIST in the development of PC still need to be further explored.

MicroRNAs (miRNAs), which bind to the 3'-untranslated region of mRNAs and regulate mRNA expression, are small non-coding RNAs (14). It has been demonstrated that miR$185-5 \mathrm{p}$ could act as a tumor-suppressive gene involved in mediating the formation of various tumor tissues, like hepatocellular carcinoma, colorectal cancer and NSCLC $(15,16)$. Consistently, miR-185-5p was down-regulated in $\mathrm{PC}$ and could also inhibit the proliferation of PC cell via targeting transcriptional co-activator $(15,17)$. Taken together, we hypothesized that miR-185-5p may also exert an anti-tumor function in PC.

We provided insights in this study into the promotive function of lncRNA XIST and the regulatory network of XIST, miR-185-5p, CCND2 in PC. Our findings suggested that lncRNA XIST could exert its oncogenic function as a ceRNA for miR-185-5p to modulate CCND2 expression, which might provide some new targets for PC treatment.

\section{Methods}

\section{Tissue samples}

PC specimens and adjacent normal tissues were collected from 70 patients who received surgery in the Second Xiangya Hospital of Central South University from 2012 to 2016. All tissues were immediately collected after resection and stored under $-80{ }^{\circ} \mathrm{C}$ after washing with sterile phosphate-buffered saline (PBS). All patients were informed to write consent and all the experiments involved in this study were approved by the Ethics Committee of the Second Xiangya Hospital of Central South University (No. 201103301).

\section{Cell culture and transfection}

PC cell lines PANC-1, ASPC-1, HPAC, BxPC-3 cells and normal pancreatic cell line HPDE cells were all purchased from ATCC (Manassas, VA, USA). Briefly, cells were cultured in RPMI 1640 medium (Thermo Fisher Scientific, USA) containing $10 \%$ Gibco ${ }^{\circledR}$ fetal bovine serum (FBS) and $100 \mu \mathrm{g} / \mathrm{mL}$ penicillin-streptomycin (Sigma-Aldrich Co, USA) at $37^{\circ} \mathrm{C}$ and $5 \% \mathrm{CO}_{2}$. Lentivirus for shRNA for XIST (sh-XIST), negative control (NC) and overexpression plasmids for XIST (pcDNA3.1-XIST) were purchased from Shanghai GenePharma Co., Ltd. (Shanghai, China). miR185-5p mimics, miR-185-5p inhibitor and their negative controls were purchased from RiboBio (Guangzhou, China). After cultured to 70-80\% confluence, cells were transfected with sh-XIST, pcDNA3.1-XIST, or miR-185$5 \mathrm{p}$ mimics, miR-185-5p negative control at a concentration of $50 \mathrm{nmol} / \mathrm{L}$ using Lipofectamine 3000 (Invitrogen, USA) according to the manufacturer's instruction.

\section{Flow cytometry assay}

Flow cytometry was performed to assess the cell cycle and apoptosis. For the detection of cell cycle, briefly, cells were trypsinized and fixed with $70 \%$ absolute ethyl alcohol overnight at $4{ }^{\circ} \mathrm{C}$. And then $100 \mu \mathrm{L}$ RNase A Reagent (Keygen Biotech, Nanjing, China) was added into cells. Propidium iodide reagent (Keygen Biotech) was used to stain the cells at the concentration of $20 \mu \mathrm{g} / \mathrm{mL}$ for 20 minutes. Then DNA content was analyzed by FACScalibur (BD Bioscience, USA). For the detection of apoptosis, cells were harvested and then re-suspended with buffer. The FITC Annexin V Apoptosis Detection 
Kit I (Ruibo, Guangzhou, China) was applied for staining the cells. A FACScan flow cytometer was performed for analysis cells apoptosis. All the experiments were in triplicated.

\section{Colony formation assay}

Cell proliferation was detected using clonogenic formation assay. Briefly, cells were seeded into a 6 -well plate at a density of 500 cells per well. The cells were fixed with $4 \%$ paraformaldehyde and stained by crystal violet $(1 \mathrm{mg} / \mathrm{mL})$ after 14 days of culture. Then the formed number of colonies in culture plate seeded with different cells was counted ( $>50$ cells) under microscope.

\section{Dual luciferase reporter assay}

To verify the prediction, dual luciferase reporter assay was conducted as reported elsewhere (18). The predicted binding mode was obtained using bioinformatic prediction by software targetscan 5.1 (http://www.targetscan. org; Whitehead Institute for Biomedical Research, Cambridge, MA, USA). PCR was performed to amplify the 3'-end fragment of lncRNA XIST containing the predicted miR-185-5p-binding site and to subclone into a pmirGLOluciferase target expression vector (Promega, USA) as the XIST wild-type vector. And the mutated miR185-5p-binding site was constructed as XIST Mutant vector. 293 T cells were co-transfected with $200 \mathrm{ng}$ of either pmirGLO-XIST-wide type (WT) or pmirGLO-XISTmutant (MUT) vector and $80 \mathrm{ng}$ of miR-185-5p mimics or inhibitor and their negative controls with Lipofectamine 3000 (Invitrogen). The verification of combination between miR-185-5p and CCND2 was realized via co-transfection with pmirGLO-CCND2-WT or pmirGLO-CCND2MUT and miR-185-5p mimics or negative control. And the relative luciferase activity was measured using Dualluciferase Reporter Assay Kit (Progema, USA) after 48 h of transfection.

\section{Quantitative real time PCR ( $q R T-P C R)$}

The expression of XIST and miR-185-5p was determined using qRT-PCR. Briefly, total RNA was extracted using Trizol reagent (Takara, Otsu, Japan) according to the manufacturer's instructions. And then, $1 \mu \mathrm{g}$ RNA was converted into cDNA using Reverse Transcription Kit (Takara). SYBR Premix Ex Taq (Takara) was applied for qRT-PCR assays. The PCR reactions were conducted in an ABI 7500 Fast RealTime PCR System (Life Technologies).

Primers used in PCR were listed below: XIST: sense: 5'-AGCTCCTCGGACAGCTGTAA-3', anti-sense: 5'-CT CCAGATAGCTGGCAACC-3'; miR-185-5p: sense: 5'-T GGAGAGAAAGGCAGTTCCTGA-3', anti-sense: 5'-GC TTCGGCAGCACATATACTAAAAT-3'; U6: sense: 5'-CT CGCTTCGGCAGCACA-3', anti-sense: 5'-AGATAGGA TTACTACAC-3' GAPDH: sense: 5'-CAAGGTCATCCA TGACAACTTTG-3', anti-sense: 5'-GTCCACCACCCT GTTGCTGTAG-3'. Relative RNA levels were calculated by the $2^{-\Delta \Delta C q}$ method. Relative expression was normalized to GAPDH and U6, respectively.

\section{Western blot analysis}

Briefly, total protein extracted from the cells was loaded on $10 \%$ SDS-PAGE and transferred to PVDF membranes (Millipore, USA). The membranes were probed with the specific primary antibodies (All purchased from Abcam, USA) as listed: CCND2 (ab207604, 1:1,000), CDC2 (ab32444, 1:1,000), CDC4 (ab12292, 1:500), CDC6 (ab109315, 1:1,000), CCND1 (ab134175, 1:10,000), Caspase-3 (ab13585, 1:500), Bcl-2 (ab32124, 1:1,000), Bax (ab32503, 1:1,000), GAPDH (ab8245, 1:500). After incubation with primary antibodies at $4{ }^{\circ} \mathrm{C}$ overnight, membranes were incubated with corresponding secondary horseradish peroxidase-conjugated anti-rabbit IgG antibody (ab6721, 1:5,000) at room temperature for $2 \mathrm{~h}$. The target bands were then scanned using Super Signal West Pico Chemiluminescent Substrate Kit (Pierce, Rockford, IL, USA). GAPDH was served as an internal control.

\section{Statistical analysis}

SPSS 18.0 and Graph Pad Prism6.0 software were used for statistical analysis. All data was presented by mean \pm Standard deviation (SD). Comparison between two groups was performed using the Student's $t$-test. Comparison among three or more groups was conducted using one-way analysis of variance (ANOVA). The correlation between XIST and miR-185-5p was determined using Spearman's analysis. It was considered to be statistically significant when $\mathrm{P}$-value was less than 0.05 . 


\section{A}

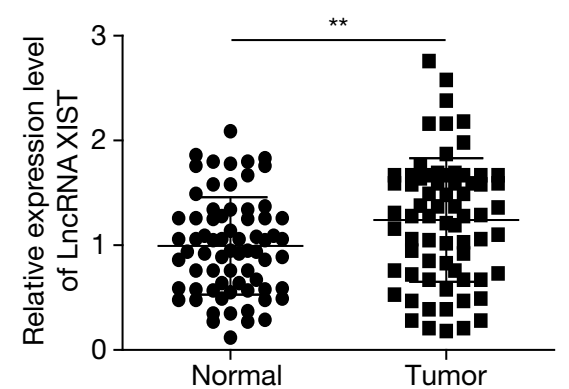

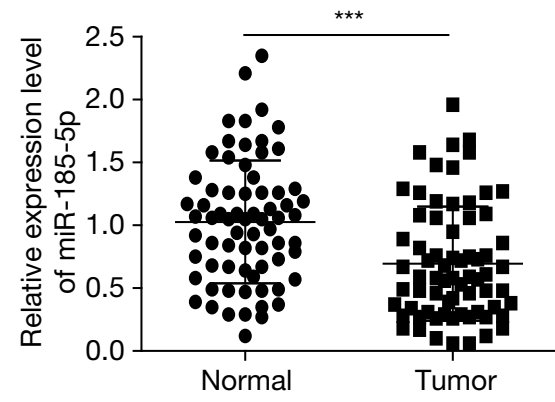
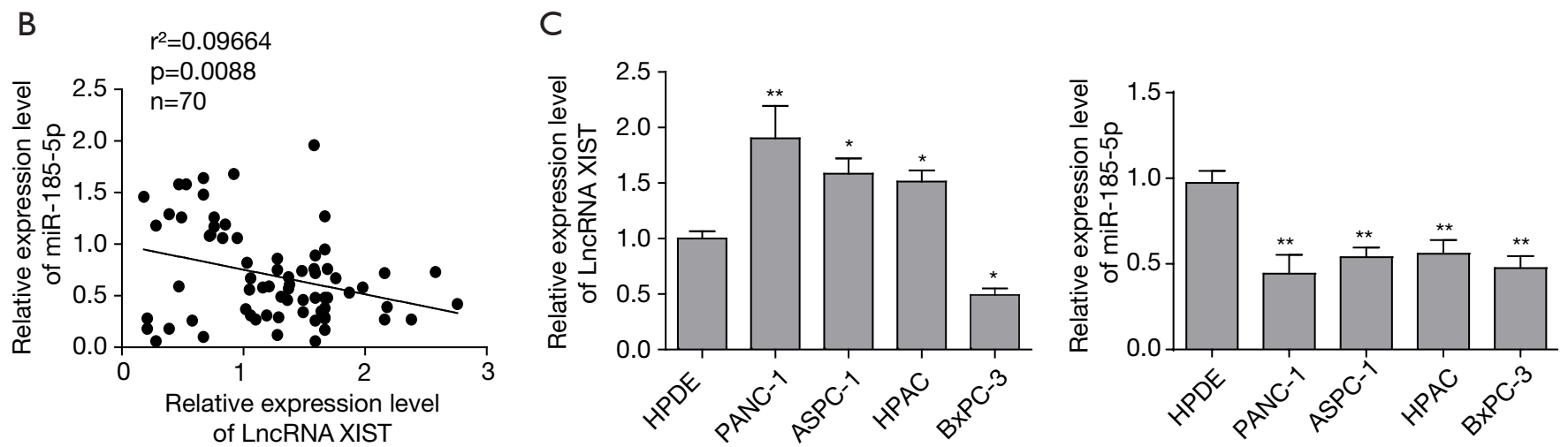

Figure 1 The expression of XIST and miR-185-5p in PC tissues and cell lines. (A) Expression of XIST and miR-185-5p in PC tissues and normal tissues. ${ }^{* *} \mathrm{P}<0.01$ and ${ }^{* * *} \mathrm{P}<0.001$ vs. normal. (B) Correlation between XIST and miR-185-5p in PC tissues by Spearman's analysis. (C) Expression of XIST and miR-185-5p in four different PC cell lines and normal pancreatic cell line HPDE cells. ${ }^{*} \mathrm{P}<0.05$ and ${ }^{* *} \mathrm{P}<0.01$ s. HPDE. XIST, X inactive-specific transcript; PC, pancreatic cancer.

\section{Results}

\section{Negative correlation between lncRNA XIST and miR-} 185-5p in PC tissues and cell lines

To determine the expression of XIST and miR-185-5p both in PC and normal tissues, firstly, we collected PC tissues and paired normal tissues from 70 patients and then the expression of XIST and miR-185-5p was measured using qRT-PCR. The results showed that lncRNA XIST was up-regulated in PC tissues, while miR-185-5p was down-regulated, compared with adjacent normal tissues (Figure 1A). Spearman's analysis also displayed negative correlation between XIST and miR-185-5p (Figure 1B). Meanwhile, four PC cell lines PANC-1, ASPC-1, HPAC, BxPC-3 cells and normal pancreatic cell line HPDE cells were applied for further confirmation. Similarly, as shown in Figure $1 C$, increasing expression of XIST and decreasing of miR-185-5p were also displayed in PC cell lines. Among all PC cells, PANC-1 cells with the highest expression of XIST and BxPC- 3 cells with the lowest expression of XIST were selected for further experiments.

\section{Effects of XIST on cell proliferation, cell cycle and apoptosis in PC cells}

To further explore whether XIST acts as an oncogene in PC, we established PANC-1 cells in which XIST was stably knocked down with sh-XIST transfection and BxPC cells in which XIST was stably overexpressed with pcDNA3.1-XIST transfection (Figure 2A), indicating the successful establishment of lncRNA XIST knockdown or overexpression cell model. Then, we observed that cell cycle was arrested in G0/G1 phase with knocking down XIST using flow cytometry assay. Whereas, cell cycle of BxPC-3 cells transfected with pcDNA3.1-XIST suggested a opposite result (Figure $2 B$ ). In parallel, colony formation assay also indicated that knockdown of XIST significantly suppressed colony formation of PANC- 1 cells, while overexpression of XIST promoted cell proliferation of BxPC-3 cells (Figure 2C). Moreover, flow cytometry 
A

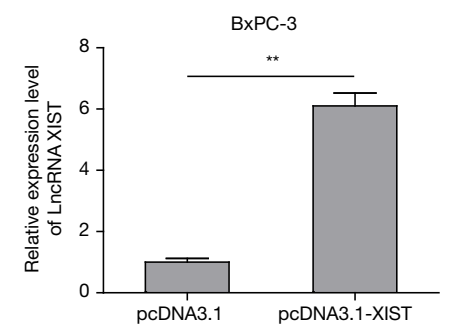

B

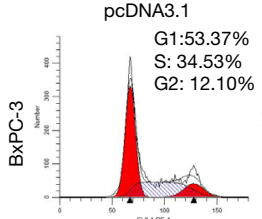

SHNC

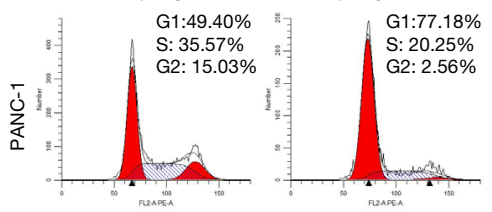

C

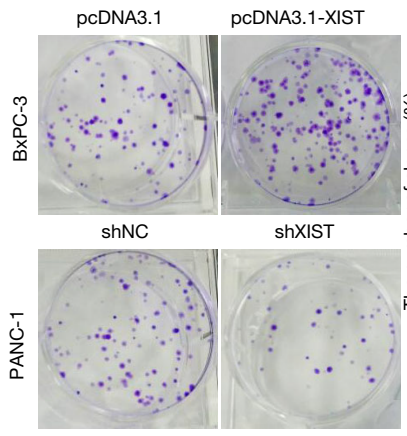

$E$

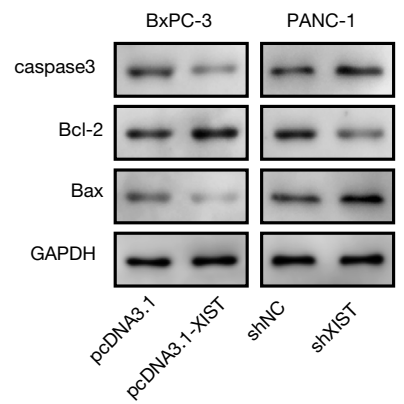

pcDNA3.1-XIST

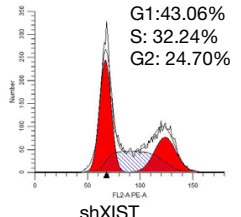
Then

$25 \%$

.
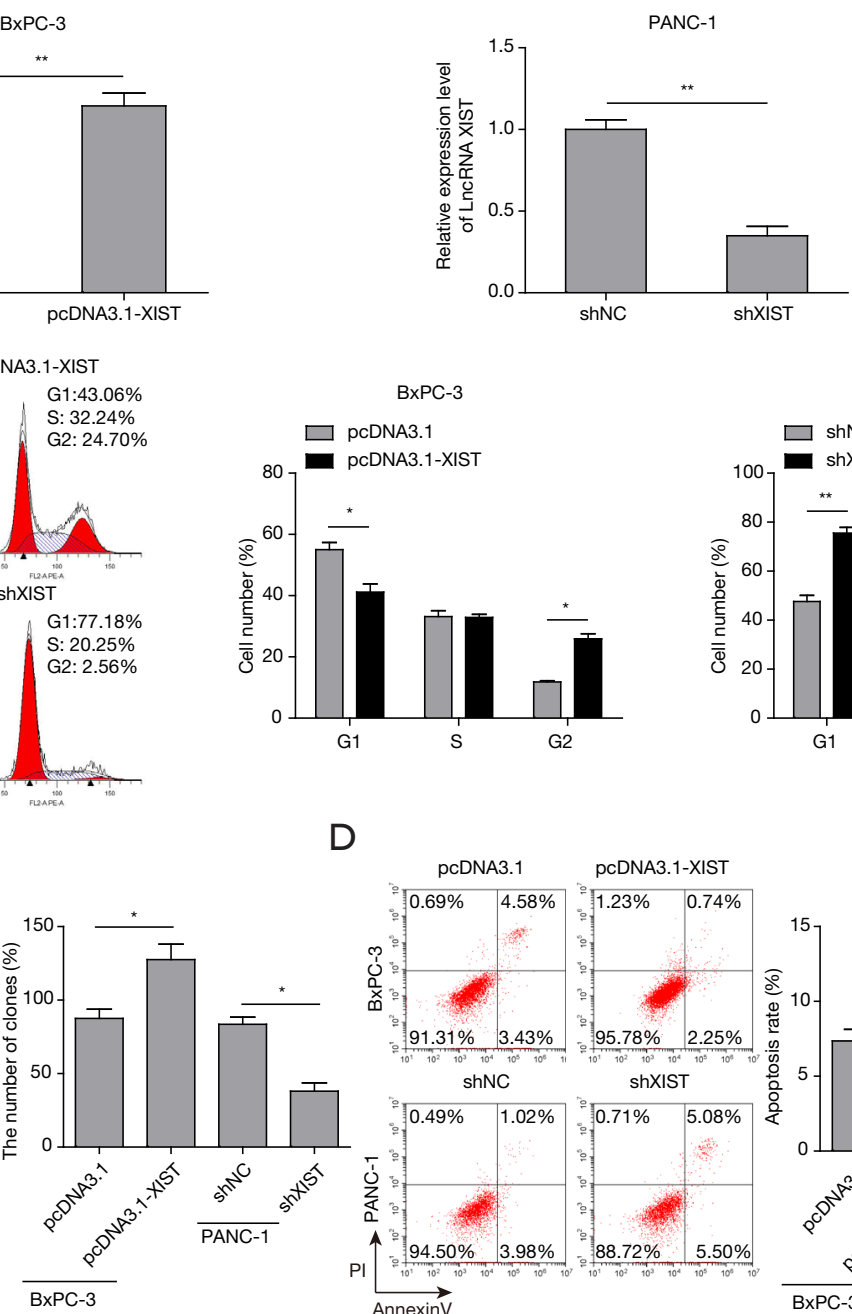

D

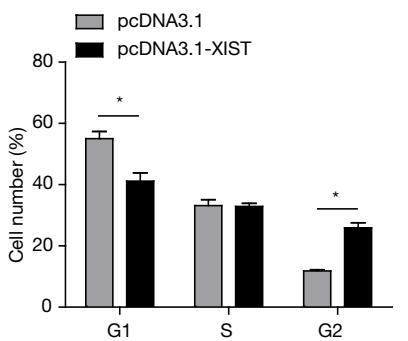

pcDNA3.1 PcDNA3.1-XIST

PANC-1
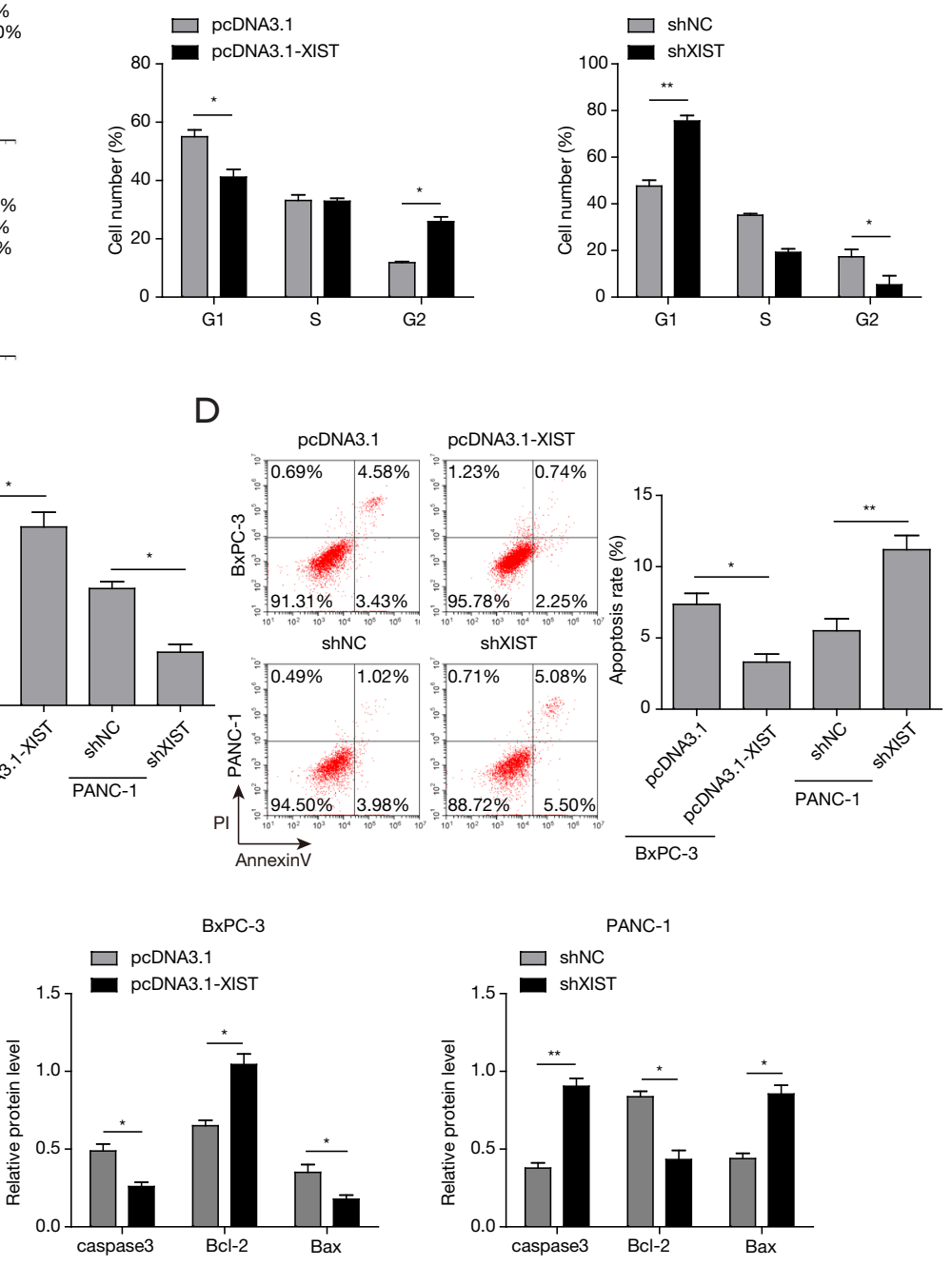

Figure 2 Effects of XIST on cells proliferation and apoptosis in BxPC-3 and PANC-1. (A) Expression of XIST in BxPC-3 cells transfected with pcDNA3.1-XIST or NC (pcDNA3.1) and in PANC-1 cells transfected with sh-XIST or shNC. ${ }^{* *} \mathrm{P}<0.01$ vs. pcDNA3.1 and ${ }^{* *} \mathrm{P}<0.01$ vs. shNC. (B) Cell cycle analysis of BxPC-3 cells transfected with pcDNA3.1-XIST or NC (pcDNA3.1) and in PANC-1 cells transfected with sh-XIST or shNC by the flow cytometry assay. ${ }^{*} \mathrm{P}<0.05 v$ s. pcDNA3.1, ${ }^{*} \mathrm{P}<0.05$ and ${ }^{* *} \mathrm{P}<0.01 v$ s. shNC. (C) Cell proliferation of BxPC3 cells transfected with pcDNA3.1-XIST or NC (pcDNA3.1) and PANC-1 cells transfected with sh-XIST or shNC by colony formation assay. ${ }^{*} \mathrm{P}<0.05$ vs. pcDNA3.1 and ${ }^{*} \mathrm{P}<0.05$ vs. shNC. (D) Cell apoptosis of BxPC-3 cells transfected with pcDNA3.1-XIST or NC (pcDNA3.1) and PANC-1 cells transfected with sh-XIST or shNC by the flow cytometry assay. ${ }^{*} \mathrm{P}<0.05$ vs. pcDNA3.1 and ${ }^{*} \mathrm{P}<0.01$ vs. shNC. (E) Western blot analysis was performed to measure the expression of Caspase-3, Bcl-2 and $\mathrm{Bax} .{ }^{*} \mathrm{P}<0.05$ vs. pcDNA3.1, ${ }^{*} \mathrm{P}<0.05$ and ${ }^{*} \mathrm{P}<0.01$ $v s$ shNC. XIST, X inactive-specific transcript. 

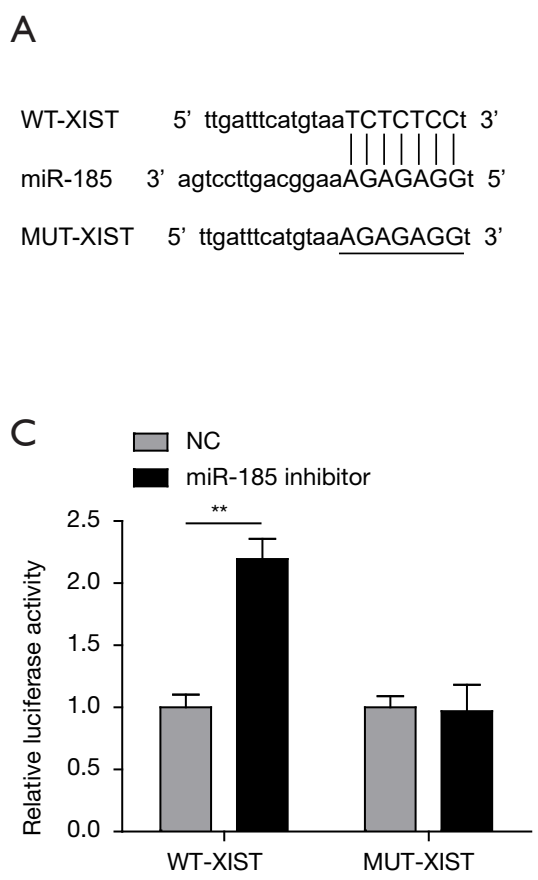
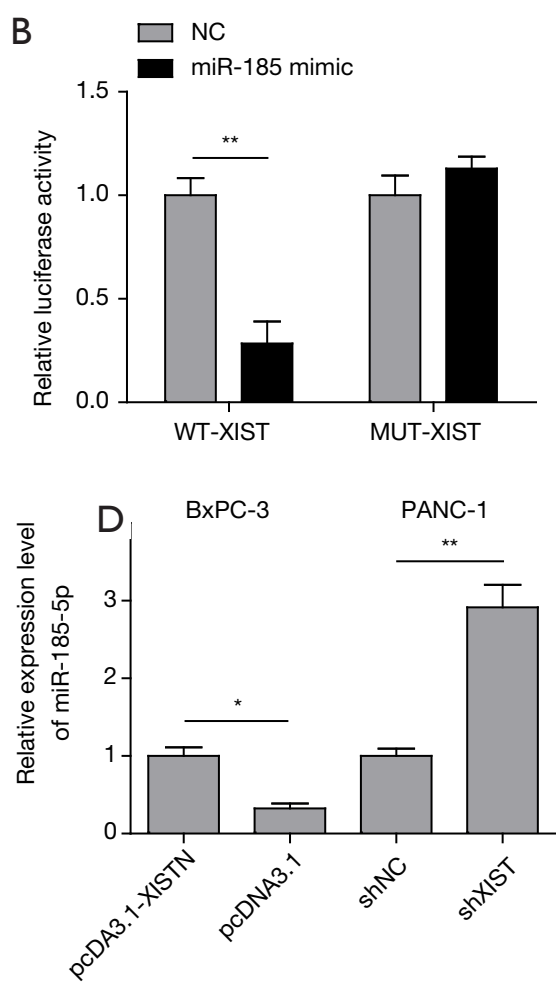

Figure 3 miR-185-5p is a target of XIST. (A) Predicted binding site of XIST and miR-185-5p. (B) and (C) Luciferase activity of WT-XIST and MUT-XIST transfected with miR-185-5p mimics, inhibitor or their negative controls (NC). ${ }^{* *} \mathrm{P}<0.01$. (D) Expression of miR-185-5p in BxPC-3 cells transfected with pcDNA3.1-XIST or NC (pcDNA3.1) and in PANC-1 cells transfected with sh-XIST or shNC. *P<0.05 vs. pcDNA3.1 and ${ }^{* *} \mathrm{P}<0.01$ vs. shNC. XIST, $\mathrm{X}$ inactive-specific transcript.

assay showed a significant increase of cell apoptosis after knockdown of XIST in PANC-1 cells, while cell apoptosis showed a reduction with overexpression of XIST in BxPC3 cells (Figure 2D). In addition, western blot analysis also indicated that overexpression of XIST induced the upregulation of Bcl-2 and down-regulation of Caspase-3, Bax, while XIST knockdown showed the opposite tendency (Figure 2E). Taken together, these data indicated that lncRNA XIST might function as an oncogene to enhance the tumorigenesis of PC.

\section{miR-185-5p is a direct target of XIST}

Recently, accumulating evidence has proved that lncRNAs may participate in the ceRNA regulatory network. Bioinformatic analysis suggested that there is the complementary base pairing between XIST and miR-185$5 \mathrm{p}$ (Figure $3 A$ ). To confirm the direct binding relationship between XIST and miR-185-5p, a luciferase reporter assay was performed. We found that miR-185-5p mimic markedly reduced the luciferase activities of pmirGLO-XIST-WT, while there was no obvious difference in cells transfected with miR-185-5p mimic and pmirGLO-XIST-MUT (Figure 3B). Consistently, the luciferase activities in cells transfected with miR-185-5p inhibitor and pmirGLOXIST-WT or pmirGLO-XIST-MUT showed the same result (Figure 3C). Additionally, an inverse correlation of XIST and miR-185-5p in PC cells was also revealed. As shown in Figure 3D, up-regulation of XIST significantly suppressed miR-185-5p expression in BxPC-3 cells, while down-regulation of XIST markedly increased miR-185$5 \mathrm{p}$ expression. These findings implied that XIST directly targets and negatively regulates $\mathrm{miR}-185-5 \mathrm{p}$.

\section{Effects of miR-185-5p on cell proliferation, cell cycle and apoptosis in PC cells}

To further investigate the potential role of miR-185-5p in PC cells, miR-185-5p was overexpressed or knocked down in both BxPC-3 and PANC-1 cells. As shown in Figure 4A, 
A
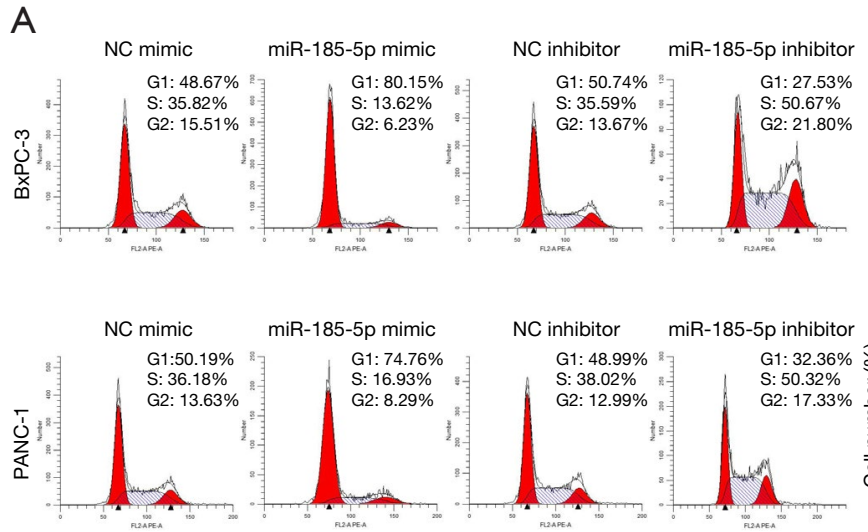

B
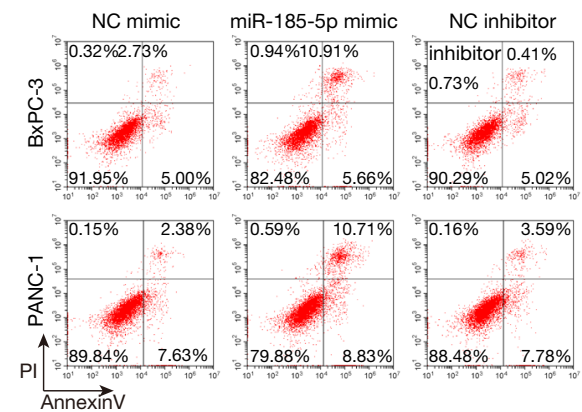

\begin{tabular}{lll} 
& & \\
& $88.48 \%$ & $7.78 \%$ \\
\hline
\end{tabular}

Annexinv

C

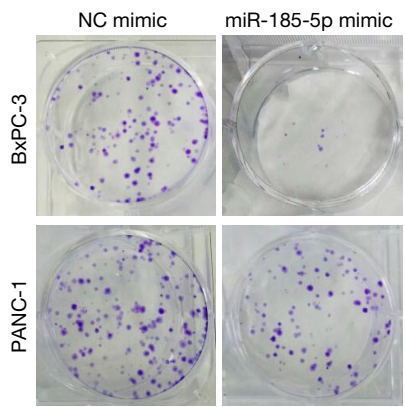

D
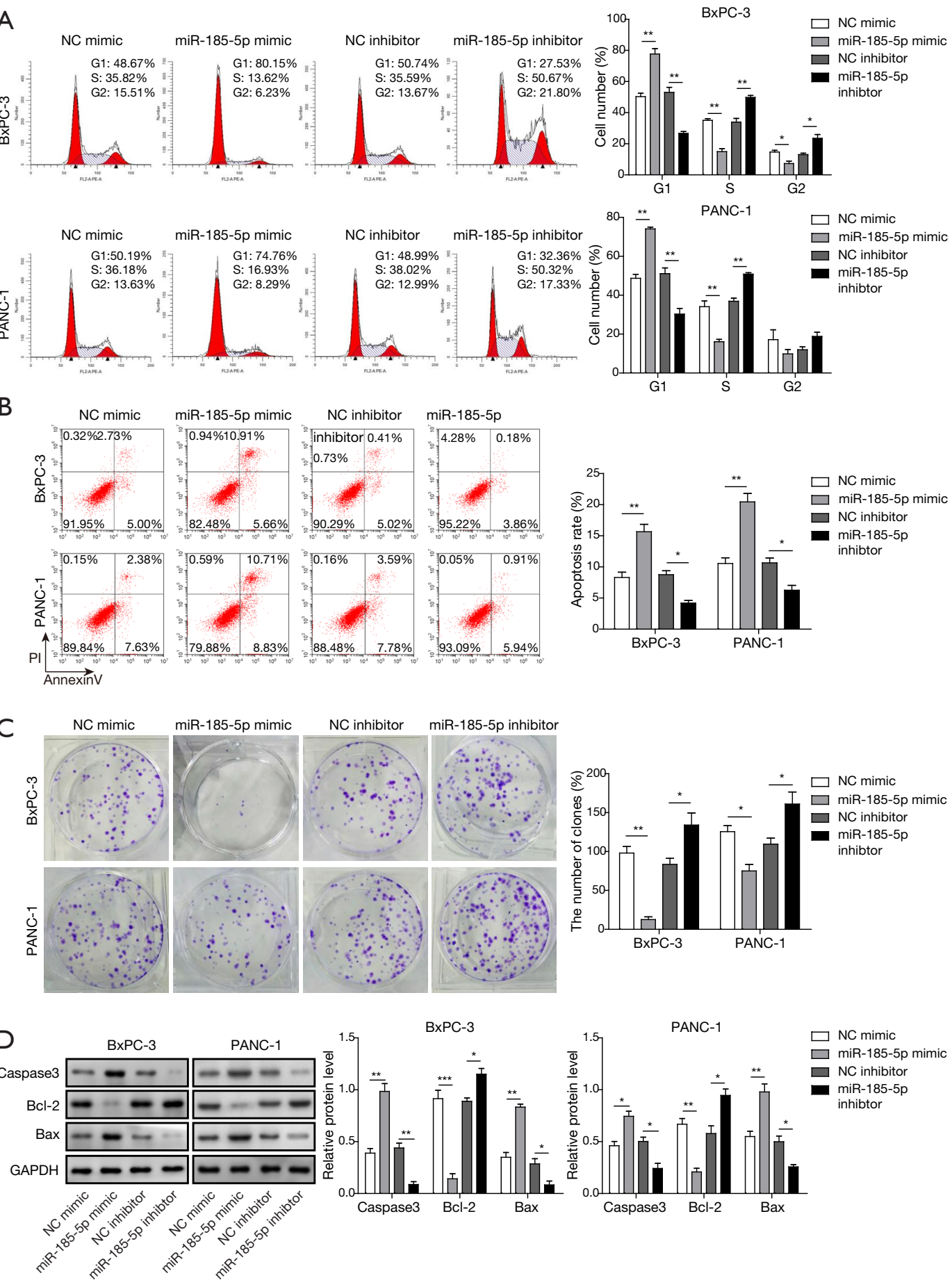

$\square$ NC mimic

miR-185-5p mimic

NC inhibitor

miR-185-5p inhibtor
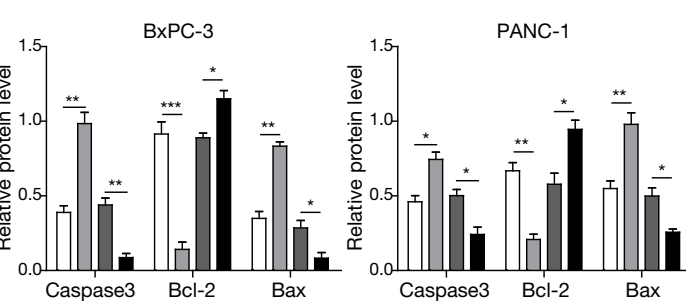

$\square$ NC mimic $\square$ miR-185-5p mimic

$\square$ NC inhibitor

miR-185-5p inhibtor

Figure 4 Effects of miR-185-5p on cell proliferation and apoptosis in BxPC-3 and PANC-1. (A) Cell cycle analysis of BxPC-3 and PANC1 cells transfected with miR-185-5p mimics, inhibitor or $\mathrm{NC}$ by flow cytometry. ${ }^{*} \mathrm{P}<0.05$ and ${ }^{* *} \mathrm{P}<0.01$ vs. $\mathrm{NC}$ mimic, ${ }^{*} \mathrm{P}<0.05$ and ${ }^{* *} \mathrm{P}<0.01$ vs. NC inhibitor. (B) Cell proliferation of BxPC-3 cells and PANC-1 cells transfected with miR-185-5p mimics, inhibitor or their negative controls (NC) by colony formation assay. ${ }^{*} \mathrm{P}<0.05$ and ${ }^{*} \mathrm{P}<0.01$ vs. NC mimic and ${ }^{*} \mathrm{P}<0.05$ vs. NC inhibitor. (C) Cell apoptosis of BxPC-3 cells and PANC-1 cells transfected with miR-185-5p mimics, inhibitor and their negative controls (NC) by the flow cytometry. ${ }^{* *} \mathrm{P}<0.01$ vs. NC mimic and ${ }^{*} \mathrm{P}<0.05$ vs. NC inhibitor. (D) Western blot analysis was performed to measure the expression of Caspase-3, Bcl-2 and Bax. ${ }^{*} \mathrm{P}<0.05,{ }^{* *} \mathrm{P}<0.01$ and ${ }^{* * *} \mathrm{P}<0.001$ vs. $\mathrm{NC}$ mimic. ${ }^{*} \mathrm{P}<0.05$ and ${ }^{* *} \mathrm{P}<0.01$ vs. $\mathrm{NC}$ inhibitor. 

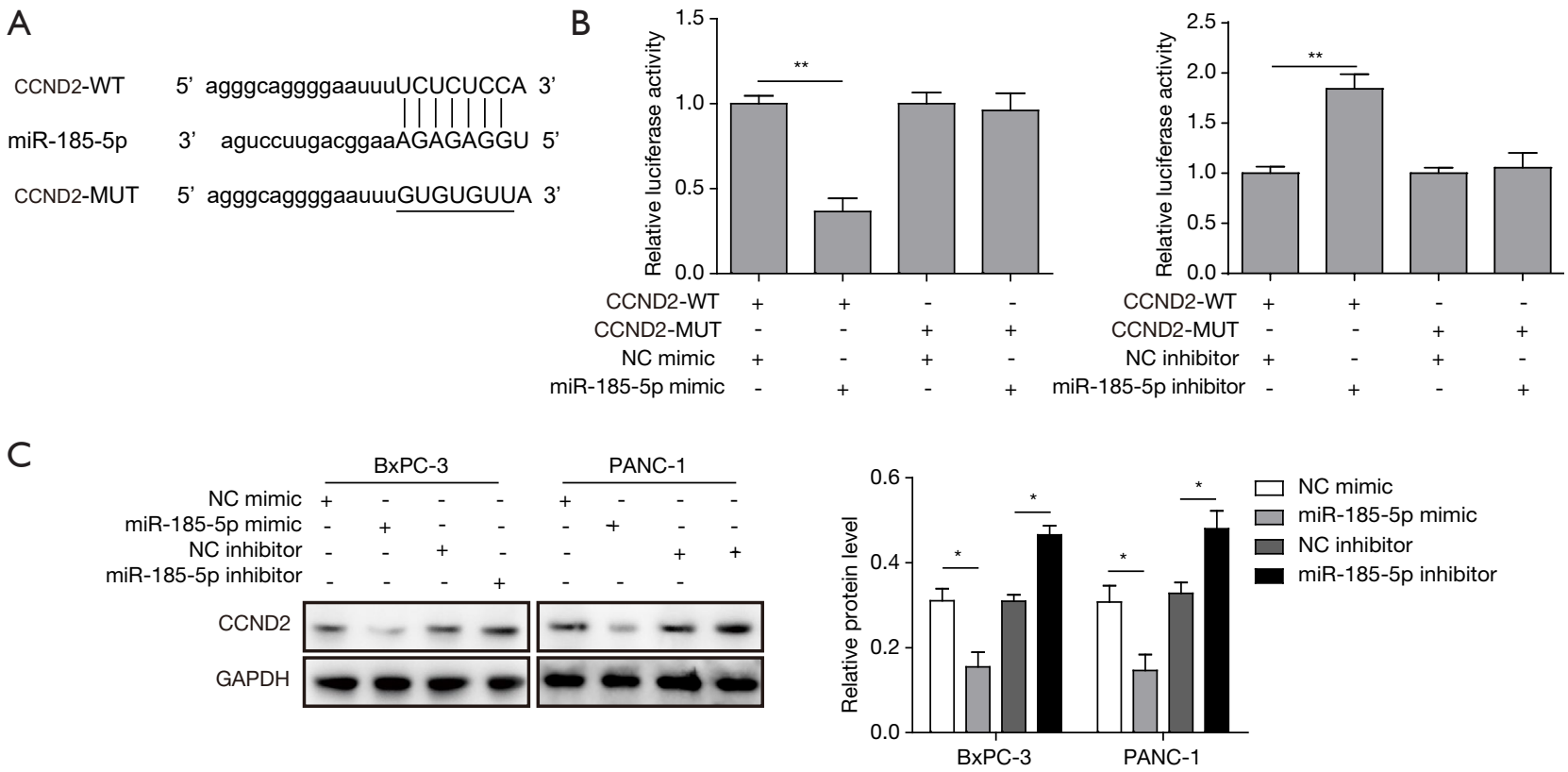

Figure 5 CCND2 is a direct target of miR185-5p. (A) Predicted binding sequence of miR-185-5p and CCND2. (B) Luciferase activity of WT-CCND2 and MUT-CCND2 transfected with miR-185-5p mimics, inhibitor or NC. ${ }^{*} \mathrm{P}<0.01$. (C) Expression of CCND2 in BxPC3 and PANC-1 cells transfected with miR-185-5p mimics, inhibitor or their negative controls (NC). ${ }^{*} \mathrm{P}<0.05 v s$. $\mathrm{NC}$ mimic and ${ }^{*} \mathrm{P}<0.05 v s$. NC inhibitor.

in both BxPC-3 and PANC-1 cells, when miR-185-5p was overexpressed, cells in G0/G1 were significantly increased and cells in G2/M and $\mathrm{S}$ were significantly decreased, while cells transfected with miR-185-5p inhibitor showed opposite results. In addition, high expression of miR-185$5 \mathrm{p}$ suppressed cell proliferation and induced apoptosis using the colony formation and flow cytometry assays, respectively. On the contrary, inhibition of miR-185-5p presented the opposite results (Figure 4B,C). Besides, the expression of Bcl2 was significantly increased within miR-185-5p inhibitor, but Caspase- 3 and Bax expression were repressed. Similarly, miR-185-5p mimic displayed the same trend (Figure 4D). All these results indicated miR-185-5p acted as a tumorsuppressive mediator in the development of PC.

\section{CCND2 is a direct target of miR-185-5p}

To study the role of miR-185-5p in the mechanisms of PC, bioinformatics analysis was performed to predict the target of miR-185-5p and found that CCND2 may be related to miR-185-5p (Figure 5A). And then the luciferase reporter assay was subjected to validate the effect of miR-185-5p on CCND2 expression. The results showed the luciferase activity was significantly decreased when cells were cotransfected with miR-185-5p mimic and CCND2 wild-type vector, but not CCND2 mutant vector, while the luciferase activity was increased within miR-185-5p inhibitor cotransfected with CCND2 wild-type vector (Figure 5B). In addition, western blot analysis was performed to measure the expression of CCND2. And the results showed that transfection with miR-185-5p mimic decreased CCND2 protein expression, while transfection with miR-185-5p inhibitor increased CCND2 expression level (Figure 5C).

\section{lncRNA XIST acts as a ceRNA sponging miR-185-5p to regulate CCND2 modulating cell proliferation and apoptosis}

In order to explore whether XIST exerted its oncogenic role through miR-185-5p/CCND2 axis in PC, cells were transfected with pcDNA3.1-XIST and miR-185-5p mimic or sh-XIST and miR-185-5p inhibitor in combination. Results showed that in BxPC-3 cells, when transfected with pcDNA3.1-XIST, cell proliferation was significantly increased, while this effect was blocked with miR-185$5 \mathrm{p}$ mimic (Figure 6A). On the contrary, in PANC- 1 cells transfected with sh-XIST, cell proliferation was significantly 
A
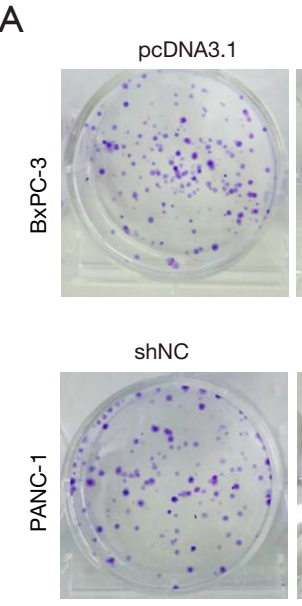

B
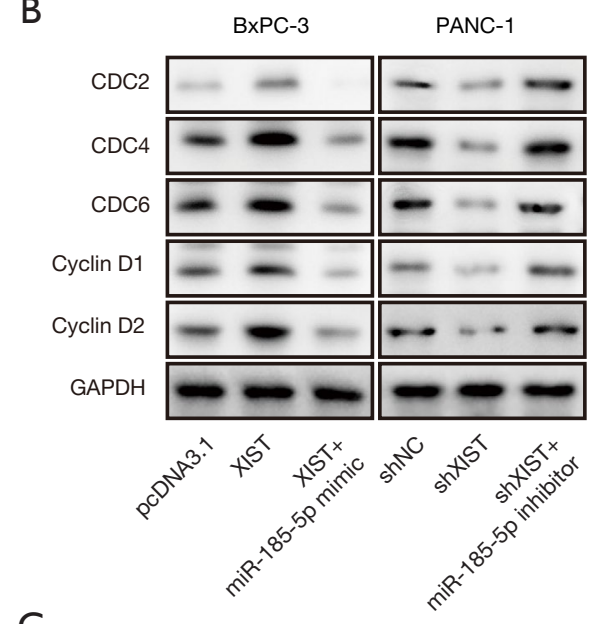

C

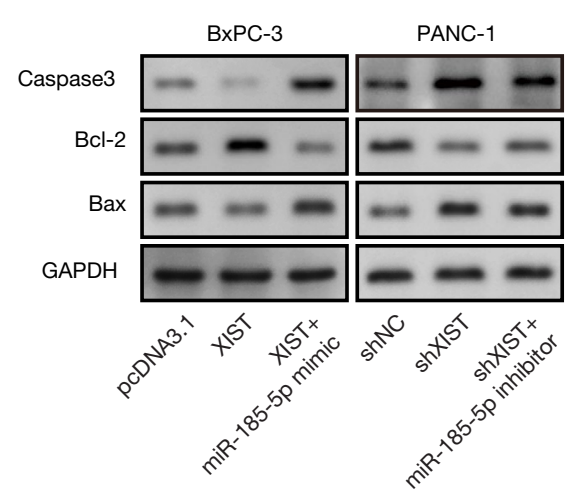

$\mathrm{XIST}+$ miR-185-5p mimic

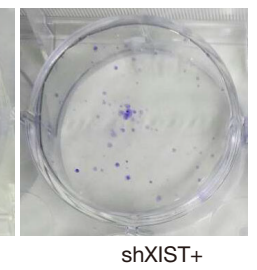
miR-185-5p inhibitor
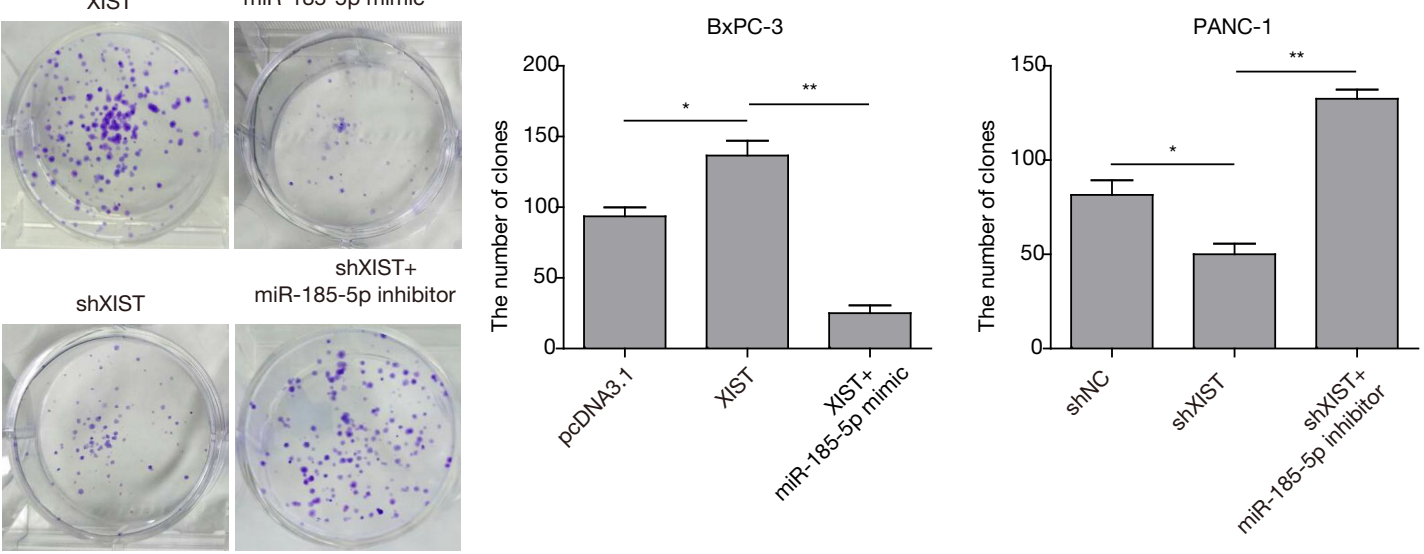
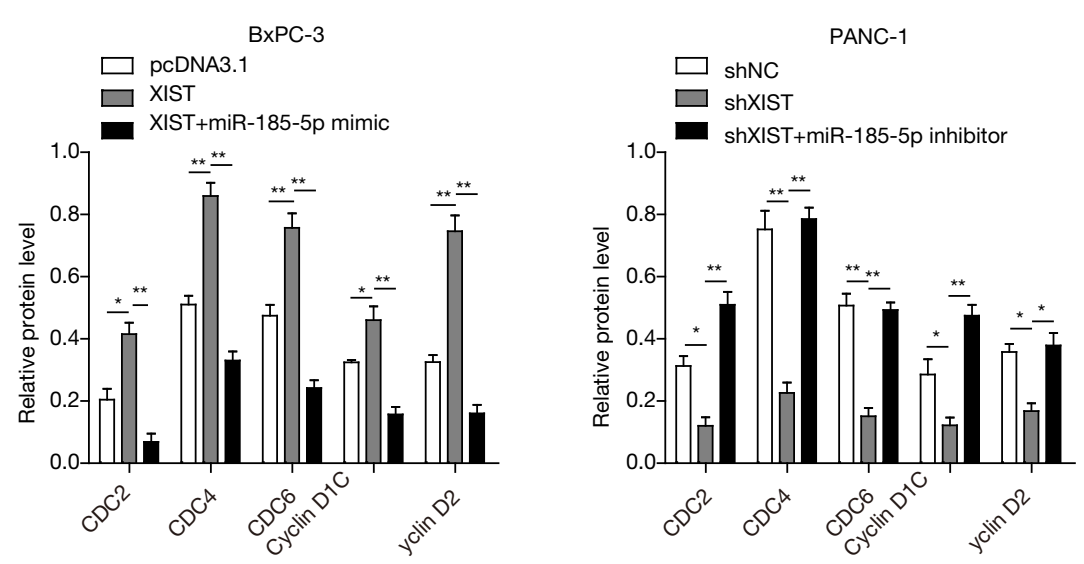

Figure 6 XIST acts as a ceRNA to regulate CCND2 via sponging miR-185-5p. (A) Cell proliferation of BxPC-3 cells transfected with pcDNA3.1, pcDNA3.1-XIST, pcDNA3.1-XIST and miR-181-5p mimics, and PANC-1 cells transfected shNC, sh-XIST, sh-XIST and miR-181-5p inhibitor by colony formation assay. ${ }^{*} \mathrm{P}<0.05$ vs. pcDNA3.1, ${ }^{*} \mathrm{P}<0.05$ vs. shNC, ${ }^{* *} \mathrm{P}<0.01$ vs. pcDNA3.1-XIST and ${ }^{* *} \mathrm{P}<0.01$ vs. sh-XIST. (B) Expression of CCND2 and cell cycle related proteins CDC2, CDC4, CDC6, CCND1 in BxPC-3 and PANC-1 cells. ${ }^{*} \mathrm{P}<0.05$ and ${ }^{* *} \mathrm{P}<0.01$ vs. pcDNA3.1, ${ }^{*} \mathrm{P}<0.05$ and ${ }^{* *} \mathrm{P}<0.01$ vs. shNC, ${ }^{* *} \mathrm{P}<0.01$ vs. pcDNA3.1-XIST, ${ }^{*} \mathrm{P}<0.05$ and ${ }^{* *} \mathrm{P}<0.01$ vs. sh-XIST. (C) Proteins expression of Caspase- $3, \mathrm{Bcl}-2$ and Bax was determined using western blot analysis. ${ }^{*} \mathrm{P}<0.05$ vs. pcDNA3.1 and ${ }^{* *} \mathrm{P}<0.01$ vs. shNC. ${ }^{*} \mathrm{P}<0.01$ and ${ }^{* * *} \mathrm{P}<0.001 v$ v. pcDNA3.1-XIST. ${ }^{*} \mathrm{P}<0.05$ vs. shNC. XIST, $\mathrm{X}$ inactive-specific transcript. 
inhibited but the effect was significantly recovered with miR-185-5p inhibitor (Figure 6A). Besides, expression levels of CCND2 and cell cycle-related proteins CDC2, CDC4, CDC6, CCND1 were significantly up-regulated when XIST was overexpression in BxPC-3 cells and were markedly down-regulated when XIST was knocked down in PANC-1 cells. And these effects were significantly reversed by overexpression or inhibition of miR-185-5p, respectively (Figure 6B). Meanwhile, overexpression of XIST, decreased the expression of Caspase- 3 and Bax, but induced Bcl2 up-regulation, and when XIST was knocked down, the alteration trends were opposite. Altogether, all these effects were reversed by additional transfection of miR-185-5p mimic or inhibitor (Figure 6C). These results suggested that miR-185-5p could be involved in XIST-mediated biological functions of PC, indicating that XIST could modulate cell proliferation and apoptosis through miR-185-5p/CCND2 axis in the development of PC.

\section{Discussion}

Despite the development of treatment and diagnosis, the prognosis of PC is still very poor and thus deeper understanding for molecular mechanisms of $\mathrm{PC}$ development is urgent (19). Currently, the dysregulation of non-coding RNAs have been demonstrated in various cancers, including PC $(20,21)$. For example, Huang et al. showed that upregulation of lncRNA PVT1 in PC patients was closely associated with the patients' poor prognosis (22). Gu et al. also found lncRNA MEG3 exerted its anti-cancer effects contributing to the suppression on PC development (23). It was also observed lncRNA HOTTIP could modulate cancer stem cell properties by regulating HOXA9 in PC (24). Additionally, lncRNA XIST was also reported to be involved in the progression of PC. A recent study demonstrated that XIST could regulate proliferation of PC cells through regulation of miR-133a/EGFR (13). However deeper insights for how XIST influence PC process are still unknown.

In the present study, we also found XIST was upregulated in PC cells. Moreover, we found that XIST contributed to cell proliferation and inhibited cell apoptosis of PC cells, and the effects might be through inhibiting miR-185-5p. Roles for XIST in PC and other cancers as an oncogene have been reported in several researches. It has been proved that up-regulation of XIST was associated with the development of PC (12). Sun et al. showed XIST exerted oncogenic functions in PC by miR-34a-5p (18). Yao et al. indicated that the inhibition of XIST showed tumorsuppressive functions in human glioblastoma stem cells by up-regulating miR-152 (25). On the contrary, several studies also revealed the anti-tumor function of XIST in the cancer development. It was found that loss of XIST could promote brain metastasis in breast cancer (26). Huang et al. also found knockdown of XIST resulted in enhanced cell viability (27). Thus, these findings suggested that XIST might demonstrate different roles in different cancers.

miR-185-5p has been proven as a tumor suppressor in several cancers. It was found in prostatic cancer tissue and cell lines, miR-185-5p was down-regulated (28). And miR-185$5 \mathrm{p}$ could also inhibit the epithelial mesenchymal transition of breast cancer cells (29). LncRNAs such as H19 and PDIA3P were also reported to regulate $\mathrm{miR}-185-5 \mathrm{p}$ in cancer development (30,31). Moreover, a recent study demonstrated XIST could promote progression of gastric cancer by targeting miR-185 (32). In the present study, we verified that miR-185-5p was also a downstream target of XIST in PC. Meanwhile, miR-185-5p was closely associated with XISTmediated biological functions in PC cells. Our results showed that XIST exerts its oncogenic functions on cell proliferation, cell cycle and apoptosis, thus contributing to PC progression, while these effects were significantly reversed by miR-185$5 \mathrm{p}$. Our finding indicated that miR-185-5p might be a tumor suppressor in the development of PC.

Role of CCND2 in cancer development has been noticed in many studies. Evron et al. demonstrated CCND2 expression was down-regulated in the majority of breast cancers (33). Zhang et al. showed miR-206 could inhibit gastric cancer proliferation by repressing CCND2 (34). Besides, several studies also showed the relationship of miR185-5p and CCND2. Sun et al. found lncRNA PDIA3P could interact with miR-185-5p by targeting CCND2 in oral squamous cell carcinoma (35). Bibaki et al. showed miR-185 could target on CCND2 in lung cancer (36). In the present study, we also found miR-185-5p could inhibit proliferation and migration of $\mathrm{PC}$ cells by down-regulation of CCND2. Besides, we confirmed that CCND2 was a downstream target of miR-185-5p, which could directly target CCND2 in PC cells, and the effects were regulated by XIST. The present study also has some limitations. First CCND2 is not the only downstream target of XIST in cancer development, thus the XIST/miR-185-5p/ $\mathrm{CCN} 2$ axis is also one of the regulation ways for PC. Other signaling pathways and deeper insights are still needed.

In conclusion, our study provided the first evidence that XIST could promote cell proliferation, inhibit cell apoptosis 
through modulating miR-185-5p/CCND2 axis. This study might give deeper understanding for role of XIST and miR$185-5 \mathrm{p}$ in development of $\mathrm{PC}$, and might provide some new targets for PC treatment. However, our study is limited in vitro experiments and the certain molecular mechanisms of XIST in PC need to be further verified in vivo.

\section{Acknowledgments}

Funding: None.

\section{Footnote}

Conflicts of Interest: All authors have completed the ICMJE uniform disclosure form (available at http://dx.doi. org/10.21037/tcr.2020.01.26). The authors have no conflicts of interest to declare.

Ethical Statement: The authors are accountable for all aspects of the work in ensuring that questions related to the accuracy or integrity of any part of the work are appropriately investigated and resolved. The study was conducted in accordance with the Declaration of Helsinki (as revised in 2013). All patients were informed to write consent and all the experiments involved in this study were approved by the Ethics Committee of the Second Xiangya Hospital of Central South University (No. 201103301).

Open Access Statement: This is an Open Access article distributed in accordance with the Creative Commons Attribution-NonCommercial-NoDerivs 4.0 International License (CC BY-NC-ND 4.0), which permits the noncommercial replication and distribution of the article with the strict proviso that no changes or edits are made and the original work is properly cited (including links to both the formal publication through the relevant DOI and the license). See: https://creativecommons.org/licenses/by-nc-nd/4.0/.

\section{References}

1. Zhang Q, Zeng L, Chen Y, et al. Pancreatic Cancer Epidemiology, Detection, and Management. Gastroenterol Res Practice 2016;2016:1-10.

2. Siegel R, Ma J, Zou Z, et al. Cancer statistics, 2014. CA Cancer J Clin 2014;64:9-29.

3. Kaistha BP, Honstein T, Müller V, et al. Key role of dual specificity kinase TTK in proliferation and survival of pancreatic cancer cells. Br J Cancer 2014;111:1780.
4. Ma L, Wang F, Du C, et al. Long non-coding RNA MEG3 functions as a tumour suppressor and has prognostic predictive value in human pancreatic cancer. Oncol Rep 2018;39:1132.

5. Tang Y, He Y, Zhang P, et al. LncRNAs regulate the cytoskeleton and related Rho/ROCK signaling in cancer metastasis. Mol Cancer 2018;17:77.

6. Duguang L, Jin $\mathrm{H}$, Xiaowei Q, et al. The involvement of lncRNAs in the development and progression of pancreatic cancer. Cancer Biol Ther 2017;18:927-36.

7. Zhou Y, Chen Y, Ding W, et al. LncRNA UCA1 impacts cell proliferation, invasion, and migration of pancreatic cancer through regulating miR-96/FOXO3. Iubmb Life 2018;70:276-90.

8. McHugh CA, Chen CK, Chow A, et al. The Xist lncRNA interacts directly with SHARP to silence transcription through HDAC3. Nature 2015;521:232.

9. Fang J, Sun CC, Gong C. Long noncoding RNA XIST acts as an oncogene in non-small cell lung cancer by epigenetically repressing KLF2 expression. Biochem Biophys Res Commun 2016;478:811-7.

10. Chen DL, Ju HQ, Lu YX, et al. Long non-coding RNA XIST regulates gastric cancer progression by acting as a molecular sponge of miR-101 to modulate EZH2 expression. J Exp Clin Cancer Res Cr 2016;35:142.

11. Yildirim E, Kirby JE, Brown DE, et al. Xist RNA Is a Potent Suppressor of Hematologic Cancer in Mice. Cell 2013;152:727-42.

12. Liang S, Gong X, Zhang G, et al. The lncRNA XIST interacts with miR-140/miR-124/iASPP axis to promote pancreatic carcinoma growth. Oncotarget 2017;8:113701.

13. Wei W, Liu Y, Lu Y, et al. LncRNA XIST Promotes Pancreatic Cancer Proliferation through miR-133a/ EGFR. J Cellul Biochem 2017;118.

14. Garzon R, Calin GA, Croce CM. MicroRNAs in Cancer. Ann Rev Med 2009;60:167-79.

15. Imam JS, Buddavarapu K, Leechang JS, et al. MicroRNA-185 suppresses tumor growth and progression by targeting the Six1 oncogene in human cancers. Oncogene 2010;29:4971-9.

16. Zhang Z, Liu X, Feng B, et al. STIM1, a direct target of microRNA-185, promotes tumor metastasis and is associated with poor prognosis in colorectal cancer. Oncogene 2015;34:4808-20.

17. Xia D, Li X, Niu Q, et al. MicroRNA-185 suppresses pancreatic cell proliferation by targeting transcriptional coactivator with PDZ-binding motif in pancreatic cancer. Exp Ther Med 2018;15:657. 
18. Sun Z, Zhang B, Cui T. Long non-coding RNA XIST exerts oncogenic functions in pancreatic cancer via miR34a-5p. Oncol Rep 2018;39:1591-600.

19. Hidalgo M. Pancreatic cancer. N Engl J Med 2010;362:1605-17.

20. Yoshida K, Toden S, Ravindranathan P, et al. Curcumin sensitizes pancreatic cancer cells to gemcitabine by attenuating PRC2 subunit EZH2, and the lncRNA PVT1 expression. Carcinogenesis 2017;38:1036-46.

21. Previdi MC, Carotenuto P, Zito D, et al. Noncoding RNAs as novel biomarkers in pancreatic cancer: what do we know? Future Oncol 2017;13:443-53.

22. Huang $\mathrm{C}, \mathrm{Yu} \mathrm{W}$, Wang $\mathrm{Q}$, et al. Increased expression of the lncRNA PVT1 is associated with poor prognosis in pancreatic cancer patients. Minerva Medica 2015;106:143.

23. Gu L, Zhang J, Shi M, et al. lncRNA MEG3 had anticancer effects to suppress pancreatic cancer activity. Biomed Pharmacother 2017;89:1269.

24. Fu Z, Chen C, Zhou Q, et al. LncRNA HOTTIP modulates cancer stem cell properties in human pancreatic cancer by regulating HOXA9. Cancer Lett 2017;410:68.

25. Yao Y, Ma J, Xue Y, et al. Knockdown of long non-coding RNA XIST exerts tumor-suppressive functions in human glioblastoma stem cells by up-regulating miR-152. Cancer Lett 2015;359:75-86.

26. Xing F, Liu Y, Wu SY, et al. Loss of XIST in Breast Cancer Activates MSN-c-Met and Reprograms Microglia via Exosomal miRNA to Promote Brain Metastasis. Cancer Res 2018;78:4316-30.

27. Huang YS, Chang CC, Lee SS, et al. Xist reduction in breast cancer upregulates AKT phosphorylation via HDAC3-mediated repression of PHLPP1 expression. Oncotarget 2016;7:43256-66.

Cite this article as: Wang YP, Huang Y, Hou T, Lu M. LncRNA XIST acts as a ceRNA sponging miR-185-5p to modulate pancreatic cancer cell proliferation via targeting CCND2. Transl Cancer Res 2020;9(3):1427-1438. doi: 10.21037/tcr.2020.01.26
28. Ostadrahimi S, Fayaz S, Parvizhamidi M, et al. Downregulation of miR-1266-5P, miR-185-5P and miR$30 \mathrm{c}-2$ in prostatic cancer tissue and cell lines. Oncol Lett 2018;15:8157-64.

29. Yin C, Yin C, Zhang G, et al. miR-185-5p inhibits F-actin polymerization and reverses epithelial mesenchymal transition of human breast cancer cells by modulating RAGE. Mol Med Rep 2018;18:2621-30.

30. Sun CC, Zhang L, Li G, et al. The lncRNA PDIA3P Interacts with miR-185-5p to Modulate Oral Squamous Cell Carcinoma Progression by Targeting Cyclin D2. Mol Ther Nucleic Acids 2017;9:100-10.

31. Wu T, Qu L, He G, et al. Regulation of laryngeal squamous cell cancer progression by the lncRNA H19/ miR-148a-3p/DNMT1 axis. Oncotarget 2016;7:11553-66.

32. Zhang Q, Chen B, Liu P, et al. XIST promotes gastric cancer (GC) progression through TGF- $\beta 1$ via targeting miR-185. J Cell Biochem 2018;119:2787-96.

33. Evron E, Umbricht CB, Korz D, et al. Loss of cyclin $\mathrm{D} 2$ expression in the majority of breast cancers is associated with promoter hypermethylation. Cancer Res 2001;61:2782-7.

34. Zhang L, Liu X, Jin H, et al. miR-206 inhibits gastric cancer proliferation in part by repressing cyclinD2. Cancer Lett 2013;332:94-101.

35. Sun CC, Zhang L, Li G, et al. The lncRNA PDIA3P Interacts with miR-185-5p to Modulate Oral Squamous Cell Carcinoma Progression by Targeting Cyclin D2. Mol The Nucleic Acids 2017;9:100.

36. Bibaki E, Tsitoura E, Koutoulaki C, et al. Expression of miR-185 targets DNA methyltransferase 1 and Cyclin D2 in IPF and lung cancer in BALF cells: Preliminary results. Eur Respir J 2015;46:PA3039. 